\title{
Oscillating Flow of Viscous Incompressible Fluid Through Sinusoidal Periodic Tube at Low Reynolds Number
}

\author{
Tithi Sikdar ${ }^{1}$, Nusrat Jahan Pinky ${ }^{1}$, Avijit Roy ${ }^{1}$, Shahid Shafayet Hossain ${ }^{2}$, Nazmul Islam ${ }^{1, ~ * ~}$ \\ ${ }^{1}$ Mathematics Discipline, Khulna University, Khulna, Bangladesh \\ ${ }^{2}$ Mathematics Department, Govt. K. C. College, Jhenaidah, Bangladesh \\ Email address: \\ tithirahaku@gmail.com (T. Sikdar), nusrat131216@gmail.com (N. J. Pinky), avijit858401@gmail.com (A. Roy), \\ shafayetku@gmail.com (S. S. Hossain),nazmul.islam@math.ku.ac.bd (N. Islam) \\ ${ }^{*}$ Corresponding author
}

\section{To cite this article:}

Tithi Sikdar, Nusrat Jahan Pinky, Avijit Roy, Shahid Shafayet Hossain, Nazmul Islam. Oscillating Flow of Viscous Incompressible Fluid Through Sinusoidal Periodic Tube at Low Reynolds Number. International Journal of Fluid Mechanics \& Thermal Sciences.

Vol. 6, No. 1, 2020, pp. 9-18. doi: 10.11648/j.ijfmts.20200601.12

Received: October 28, 2019; Accepted: November 28, 2019; Published: January 7, 2020

\begin{abstract}
The flow in tubes with periodically varying cross-section has many interests due to its various practical applications such as it can be used as particle separation devices. In this paper, we have examined the oscillatory flow of a viscous incompressible fluid in a sinusoidal periodic tube at low Reynolds number. The numerical study is undertaken to examine fluid movement at different cross-sections for different time. The boundary element method (BEM) has been formulated for the infinite sinusoidal periodic tube to solve the governing equations for obtaining components of surface force on the tube wall. We have calculated the axial and radial velocities at different cross-sections for different time and compared them. We find that the behaviors of the velocity curves for different cross-sections remain the same for the same phase of time over the oscillation. On the contrary, the behavior of the velocity curves become different for different phase of time. For the tube geometry, the axial velocity at the converging and diverging regions are the same while the radial velocity at these regions are the same in magnitudes but in opposite direction. In addition, the radial velocity is maximum in the half way between the tube axis and the tube wall, and it is minimum on the tube axis and on the tube wall. The obtained velocity indicates that the net fluid movement after each complete oscillation is zero, which is an assumption to separate particles in such periodic tube.
\end{abstract}

Keywords: Oscillating Flow, Low Reynolds Number, Boundary Element Method, Sinusoidal Periodic Tube

\section{Introduction}

The study of flow in tubes with periodic variations has attracted much attention of researchers due to its various physiological and engineering applications. It helps us to understand the characteristics of the blood flow through arteries or the transport of intestinal fluid through the colon [1-3]. Other applications include the investigation of transport processes in porous media and separate particle dispersions within micro fluidic devices and nano porous membranes [4-6].

A number of authors have investigated oscillating flow through different periodic tubes. Previous studies on the oscillatory flow in nonuniform channels and tubes with smooth constrictions began with Bellhouse et al. [7] who studied oscillatory flow in a furrowed channel and reported that the oscillatory flow could improve the mass transfer rate in a blood oxygenator. They confirmed that the combination of a large laminar oscillation with a much smaller mean flow through a wavy-walled channel could achieve a higher mass transfer rate. Sobey $[8,9]$ simulated the flow patterns using a two-dimensional numerical simulation for the oscillatory flow in the furrowed channel and showed that vortex formation and ejection were responsible for the enhanced mixing and mass transfer. This simulation was supported by experimental observations by Stephanoff et al. [10]. Nishimura et al. [11] conducted an experiment about the flow characteristics in wavy-walled channels for steady flow and indicated that the diverse flow structures in this geometry depended on both oscillatory Reynolds number and Strouhal number. They got the 
critical Reynolds numbers which is 350 for the wavy channel and claimed that the unsteady vortex motion significantly promotes wall shear stress at the maximum cross section. Then, Nishimura et al. [12] finished another study on mass transfer characteristics for pulsatile flow. They found that the mass transfer enhancement depends on three parameters: net flow Reynolds number, oscillatory fraction of the flow rate, and Strouhal number. Meanwhile, Ralph [13] studied the flow patterns in a sinusoidal wavywalled tube using two-dimensional simulation and experiments, and found a secondary flow separation and time-asymmetric flows in a range of Strouhal numbers. Furthermore, Lee et al. [14] discussed the mass transfer enhancement in an axisymmetric wavy channel for pulsatile laminar flow. Their numerical results indicated that the optimal Strouhal number increases as the Reynolds number and the channel wavelength decreasing. Later, Nishimura et al. [15] described the fluid flow and mass transfer with a wavy-walled tube at moderate Reynolds numbers. Taylor and Gerrard [1] presented a mathematical model to analyze the blood flow through arteries and expressed the different pressure radius relationships for elastic tube. Chakravarty and Sen [16] studied the blood flow through bifurcated artery. They solved the Navier-Stokes equation and obtained velocities at different time stages.

Recently, Islam, et al. [17] studied the recirculation flow through an infinite periodic sinusoidal, parabolic, triangular tube profile including asymmetric one and provided some comments on the recirculation volume based on the points of zero tangential force on the tube wall. But they didn't study the oscillating flow through such tube profiles, for example sinusoidal periodic tube. There is still needed further works on the oscillatory flow through sinusoidal periodic tube.

In this paper, we have investigated the oscillatory creeping flow of viscous incompressible fluid through an axi-symmetric sinusoidal periodic tube to find the characteristics of the velocity and movement of fluid particle at different cross-sections and at different time. We have used the boundary element technique to solve the governing equations and to find the effect of oscillating flow on velocity in axi-symmetric sinusoidal tube, and to reexamine the assumption that there is no net fluid movement after a complete oscillatory flow which is a postulate that use in particle separation technique.

\section{Mathematical Formulation}

Consider the creeping flow of an incompressible Newtonian fluid through an infinite axisymmetric periodic tube. An infinite sinusoidally constricted capillary is considered so that we have used pressure difference boundary condition. The flow is governed by a time-varying pressure gradient $\Delta P(t) / L$, where $\Delta P(t)$ is the instantaneous pressure drop across the wave section of the tube and $\mathrm{L}$ is the wavelength of one wave-section of the tube.

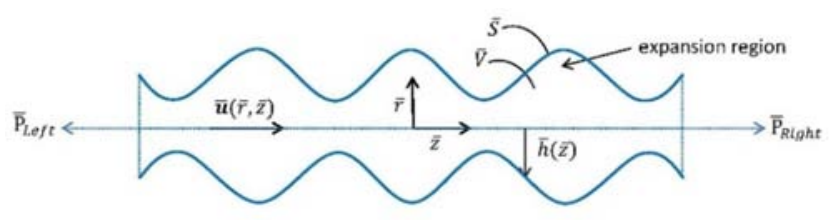

Figure 1. Schematic view of an infinitely long, cylindrical, periodically constricted capillary $h(z)=h(z+k L)$.

The surface of the tube is $\boldsymbol{y}=z \hat{\mathbf{z}}+h(z) \hat{\mathbf{r}}$, where $\hat{\mathbf{r}}$ and $\hat{\mathbf{z}}$ are unit vectors in the radial and longitudinal directions, respectively, $h(z)$ defines the tube profile. The governing equations in the absence of inertial effects (small Reynolds number, Stokes Flow) can be written in dimensional variables as

$$
\nabla^{2} \mathbf{u}=\frac{1}{\mu} \nabla \mathbf{p}, \nabla \cdot \mathbf{u}=0
$$

where $\mathbf{u}(r, z)$ is the fluid velocity, $\mathbf{p}(r, z)$ is the fluid pressure and $\mu$ is the viscosity of the fluid.

For an axisymmetric problem, we have $\nabla=\frac{\partial}{\partial r} \hat{\mathbf{r}}+\frac{\partial}{\partial z} \hat{\mathbf{z}}$.

We have considered no slip boundary conditions on the tube surface $S$, so that

$\mathbf{u}(\mathbf{x})=0$ for $\mathbf{x} \in S$.

The fundamental solutions of Stokes Equation [18] is

$$
\begin{aligned}
& \mathbf{u}(\mathbf{x})=\frac{1}{4 \pi} \int_{S} d S(\mathbf{y}) \mathbf{G}(\mathbf{x}, \mathbf{y}) \cdot \mathbf{F}(\mathbf{y})-\frac{1}{4 \pi} \int_{S} d S(\mathbf{y}) \mathbf{H}(\mathbf{x}, \mathbf{y}) \cdot \mathbf{u}(\mathbf{y}) \\
& \mathbf{u}(\mathbf{x})=\frac{1}{8 \pi} \int_{S} d S(\mathbf{y}) \mathbf{G}(\mathbf{x}, \mathbf{y}) \cdot \mathbf{F}(\mathbf{y})-\frac{1}{4 \pi} \int_{S} d S(\mathbf{y}) \mathbf{H}(\mathbf{x}, \mathbf{y}) \cdot \mathbf{u}(\mathbf{y})
\end{aligned}
$$

where, $d S$ is the surface area element of the boundary $S$ at $\mathbf{y}$ and $V$ is the interior region of the tube. In addition, $\mathbf{F}(\mathbf{y})=\sum(\mathbf{y}) \cdot \hat{\mathbf{n}}(\mathbf{y})$ is the force per unit area exerted on the fluid by the boundary at $y$ (boundary force), where $\sum(\mathbf{y})$ is the stress tensor which is defined as $\sum_{i j}=-p \delta_{i j}+\left(\frac{\partial u_{i}}{\partial x_{j}}+\frac{\partial u_{j}}{\partial x_{i}}\right)$ and the normal vector, $\hat{\mathbf{n}}(\mathbf{y})=\left(n_{r}, n_{z}\right)$ is directed outward from the control volume, $V$. Also $\mathbf{G}(\mathbf{x}, \mathbf{y})$ and $\mathbf{H}(\mathbf{x}, \mathbf{y})$ are functions of the sample point $\boldsymbol{x}$ and source point $\boldsymbol{y}$ which are defined as follows

$$
G_{i j}(\mathbf{x}, \mathbf{y})=\frac{\delta_{i j}}{\epsilon}+\frac{\hat{x}_{i} \hat{x}_{j}}{\epsilon^{3}} \text { and } H_{i j}(\boldsymbol{x}, \boldsymbol{y})=-6 \frac{\hat{x}_{i} \hat{x}_{j} \hat{x}_{k}}{\epsilon^{5}} n_{k}
$$

where, $\in=|\hat{\mathbf{x}}|$ and $\hat{\mathbf{x}}=\mathbf{y}-\mathbf{x}$.

In this study, we are particularly interested in the investigation of characterizing velocity in the constriction and expansion regions along with converging and the diverging regions of the tube due to oscillating fluid flow in the tube. To create oscillating pressure profile, we have considered the time-varying pressure difference as, 
$\Delta P(t)=P_{0} \sin (\omega \bar{t}), 0 \leq \bar{t} \leq T$, where $\quad \omega=\frac{2 \pi}{T} \quad$ is prescribed angular frequency and $T$ is the corresponding period.

\section{Numerical Computation}

We have used Boundary Element Method (BEM) to solve for the flow in the tube. The advantage of this technique is that discretisation need only be performed over the boundary (not over the whole domain) since the velocity field can be written in terms of integrals over the boundary (as in Eqs. (1) - (2)). We consider an infinitely long tube and formulate the boundary element equations over the entire length of the tube. In this case, the velocity profile at the end crosssections of the tube does not influence the calculation. The boundary element equation (1) can be solved in one representative wave section to calculate the force on the tube surface, $\mathbf{F}(\mathbf{x})$. Once this force is known, the tangential force on the tube surface, $F_{t}$ can be calculated and the fluid velocity can be calculated anywhere within the tube using equation (2). Since the tube is periodic, this one wave section gives a representation of the flow in each section of the tube.

We introduce a periodic component of the boundary force by writing

$$
\mathbf{F}\left(\mathbf{y}^{\prime}\right)=z^{\prime} \hat{\mathbf{n}}\left(\mathbf{y}^{\prime}\right)+\mathbf{f}\left(\mathbf{y}^{\prime}\right)
$$

where

$$
\mathbf{F}\left(\boldsymbol{y}^{\prime}\right)=\mathbf{f}\left(h\left(z^{\prime}\right) \hat{\mathbf{r}}, z^{\prime} \hat{\mathbf{z}}\right)=\mathbf{f}(h(z) \hat{\mathbf{r}},(z+k) \hat{\mathbf{z}})
$$

is periodic over the length of the tube $(k \in Z)$. Here, the fluid velocity on the surface of tube is zero, $\mathbf{u}(\mathbf{x})=\mathbf{0}$ for $\mathbf{x} \in S$ and for the long periodic tube, the term $G_{i, j}$ in equation (3) approach zero. Here, we consider axisymmetric tube shapes. That's why we have to reduce the surface integrals in Equation (1) and (2) into line integrals by performing the azimuthal integration analytically in a cylindrical coordinate system. For an infinite periodic tube, equation (1) can be written as

$$
\begin{aligned}
& \mathbf{u}(\mathbf{x})= \frac{1}{4 \pi} \int_{l(-K-1 / 2)}^{l(K+1 / 2)} d l\left(\mathbf{y}^{\prime}\right) \mathbf{M}\left(\mathbf{x}, \mathbf{y}^{\prime}\right) \cdot \mathbf{F}\left(\mathbf{y}^{\prime}\right) \\
&+\frac{1}{4 \pi} \int_{0}^{h(-1 / 2)} d r \mathbf{M}\left(\mathbf{x},-\left(K+\frac{1}{2}\right) \hat{\mathbf{z}}+r \hat{\mathbf{r}}\right) \cdot \mathbf{F}\left(-\left(K+\frac{1}{2}\right) \hat{\mathbf{z}}+r \hat{\mathbf{r}}\right) \\
&+\frac{1}{4 \pi} \int_{0}^{h((1 / 2)} d r \mathbf{M}\left(\mathbf{x},\left(K+\frac{1}{2}\right) \hat{\mathbf{z}}+r \hat{\mathbf{r}}\right) \cdot \mathbf{F}\left(\left(K+\frac{1}{2}\right) \hat{\mathbf{z}}+r \hat{\mathbf{r}}\right) \\
&-\frac{1}{4 \pi} \int_{l(-K-1 / 2)}^{l(K+1 / 2)} d l\left(\mathbf{y}^{\prime}\right) \mathbf{Q}\left(\mathbf{x}, \mathbf{y}^{\prime}\right) \cdot \mathbf{u}\left(\mathbf{y}^{\prime}\right) \\
&-\frac{1}{4 \pi} \int_{0}^{h(1 / 2)} d r \mathbf{Q}\left(\mathbf{x},-\left(N+\frac{1}{2}\right) \hat{\mathbf{z}}+r \hat{\mathbf{r}}\right) \cdot \mathbf{u}\left(-\left(N+\frac{1}{2}\right) \hat{\mathbf{z}}+r \hat{\mathbf{r}}\right) \\
&-\frac{1}{4 \pi} \int_{0}^{h(1 / 2)} d r \mathbf{Q}\left(\mathbf{x},\left(N+\frac{1}{2}\right) \hat{\mathbf{z}}+r \hat{\mathbf{r}}\right) \cdot \mathbf{u}\left(\left(N+\frac{1}{2}\right) \hat{\mathbf{z}}+r \hat{\mathbf{r}}\right)
\end{aligned}
$$

where $\mathbf{M}$ and $\mathbf{Q}$ are related to $\mathbf{G}$ and $\mathbf{H}$ through an angular integration as described in Chapter 2 of Pozrikidis [18], and where $\mathbf{x}\left(r_{0}, z_{0}\right)$ is a fixed point located right on the boundary in the zeroth wave-section of the capillary, $\mathbf{y}^{\prime}\left(z^{\prime}, r^{\prime}\right)=k \hat{\mathbf{z}}+\mathbf{y}$ is the variable point on boundary in the $k^{\text {th }}$ wave section of the capillary, $\mathbf{y}(z, r)$ is the variable point on the boundary in the zeroth wave section of the capillary, $d l$ is the differential arc length of the trace of the boundary and $l(z)=\int_{0}^{z} \sqrt{\left[h^{\prime}(\epsilon)\right]^{2}+1}$.

The contributions from the 4 th, 5 th and 6 th terms in the right hand side of equation (5) are zero, since the velocity is zero on the tube surface and the function $\mathbf{Q}(\mathbf{x}, \mathbf{y})$ approaches zero at the end cross-sections for a long tube. Using equation (4) into equation (5), we can write the $z$-component velocity equation as follows:

$$
\begin{aligned}
0 & =4 \pi u_{z}(\mathbf{x})=\int_{l(-1 / 2)}^{l(1 / 2)} d l(\mathbf{y}) \mathcal{M}_{z z}(\mathbf{x}, \mathbf{y})\left[z n_{z}(\mathbf{y})+f_{z}(\mathbf{y})\right]+\int_{l(-1 / 2)}^{l(1 / 2)} d l(\mathbf{y}) \mathcal{N}_{z z}(\mathbf{x}, \mathbf{y}) n_{z}(\mathbf{y}) \\
& +\int_{l(-1 / 2)}^{l(1 / 2)} d l(\mathbf{y}) \mathcal{M}_{z r}(\mathbf{x}, \mathbf{y})\left[z n_{r}(\mathbf{y})+f_{r}(\mathbf{y})\right]+\int_{l(-1 / 2)}^{l(1 / 2)} d l(\mathbf{y}) \mathcal{N}_{z r}(\mathbf{x}, \mathbf{y}) n_{r}(\mathbf{y})+4 \pi h^{2}\left(\frac{1}{2}\right)
\end{aligned}
$$

where the last term in the right hand side of equations (6) comes from the 2 nd and 3rd terms in the right hand side of equations (5), and where

$$
\begin{aligned}
& \mathcal{M}_{z z}(\mathbf{x}, \mathbf{y})=M_{z z}(\mathbf{x}, \mathbf{y})+\sum_{k=1}^{K}\left[M_{z z}(\mathbf{x}, k \hat{\mathbf{z}}+\mathbf{y})+M_{z z}(\mathbf{x},-k \hat{\mathbf{z}}+\mathbf{y})\right], \mathcal{N}_{z z}(\mathbf{x}, \mathbf{y})=\sum_{k=1}^{K} k\left[M_{z z}(\mathbf{x}, k \hat{\mathbf{z}}+\mathbf{y})-M_{z z}(\mathbf{x},-k \hat{\mathbf{z}}+\mathbf{y})\right] \\
& \mathcal{M}_{z r}(\mathbf{x}, \mathbf{y})=M_{z r}(\mathbf{x}, \mathbf{y})+\sum_{k=1}^{K}\left[M_{z r}(\mathbf{x}, k \hat{\mathbf{z}}+\mathbf{y})+M_{z r}(\mathbf{x},-k \hat{\mathbf{z}}+\mathbf{y})\right], \mathcal{N}_{z r}(\mathbf{x}, \mathbf{y})=\sum_{k=1}^{K} k\left[M_{z r}(\mathbf{x}, k \hat{\mathbf{z}}+\mathbf{y})-M_{z r}(\mathbf{x},-k \hat{\mathbf{z}}+\mathbf{y})\right]
\end{aligned}
$$


where the $M_{i j}$ are defined in Chapter 2 of Pozrikidis [18]. Since $M \sim \frac{1}{k}$ for large $\mathrm{k}[18]$, care must be taken in interpreting the sums in equation (7). By considering a force balance over one wave section of the tube and determining the behavior of the integrals in (6) at large $\mathrm{k}$, we find that for large $\mathrm{k}$

$$
\sum_{k=1}^{N}\left\{\frac{8 \pi}{k} \int_{l(-1 / 2)}^{l(1 / 2)} d l(y)\left[z n_{z}(z)+f_{z}(y)\right] h(z)-\frac{8 \pi}{k} \int_{l(-1 / 2)}^{l(1 / 2)} d l(y)\left(z-z_{0}\right) h(z) n_{z}(z)+\frac{4 \pi}{k} \int_{l(-1 / 2)}^{l(1 / 2)} d l(y) h^{2}(z) n_{r}(z)\right\}=0
$$

Subtracting Eq. (8) from Eq. (6), we obtain

$$
\begin{gathered}
u_{z}(\mathbf{x})=\frac{1}{4 \pi}\left[\int_{l(-1 / 2)}^{l(1 / 2)} d l(\mathbf{y}) \mathcal{M}_{z z}^{\prime}(\mathbf{x}, \mathbf{y})\left[z n_{z}(\mathbf{y})+f_{z}(\mathbf{y})\right]+\int_{l(-1 / 2)}^{l(1 / 2)} d l(\mathbf{y}) \mathcal{N}_{z z}^{\prime}(\mathbf{x}, \mathbf{y}) n_{z}(\mathbf{y})++\int_{l(-1 / 2)}^{l(1 / 2)} d l(\mathbf{y}) \mathcal{M}_{z r}(\mathbf{x}, \mathbf{y})\left[z n_{r}(\mathbf{y})+f_{r}(\mathbf{y})\right]\right. \\
\left.+\int_{l(-1 / 2)}^{(l / 2)} d l(\mathbf{y}) \mathcal{N}_{z r}^{\prime}(\mathbf{x}, \mathbf{y}) n_{r}(\mathbf{y})+4 \pi h^{2}\left(\frac{1}{2}\right)\right]
\end{gathered}
$$

where $\mathcal{M}_{z r}(\mathbf{x}, \mathbf{y})$ is defined above and $\mathcal{M}_{z z}^{\prime}(\mathbf{x}, \mathbf{y}), \mathcal{N}_{z z}^{\prime}(\mathbf{x}, \mathbf{y})$ and $\mathcal{N}_{z r}^{\prime}(\mathbf{x}, \mathbf{y})$ are defined below and now converge like $\frac{1}{k^{3}}$.

$$
\begin{gathered}
\mathcal{M}_{z z}^{\prime}(\mathbf{x}, \mathbf{y})=M_{z z}(\mathbf{x}, \mathbf{y})+\sum_{k=1}^{K}\left[M_{z z}(\mathbf{x}, k \hat{\mathbf{z}}+\mathbf{y})+M_{z z}(\mathbf{x},-k \hat{\mathbf{z}}+\mathbf{y})-\frac{8 \pi h(z)}{k}\right], \sum_{k=1}^{K} k\left[M_{z z}(\mathbf{x}, k \hat{\mathbf{z}}+\mathbf{y})-M_{z z}(\mathbf{x},-k \hat{\mathbf{z}}+\mathbf{y})+\frac{8 \pi\left(z-z_{0}\right) h(z)}{k^{2}}\right] \\
\mathcal{N}_{z r}^{\prime}(\mathbf{x}, \mathbf{y})=\sum_{k=1}^{K} k\left[M_{z r}(\mathbf{x}, k \hat{\mathbf{z}}+\mathbf{y})-M_{z r}(\mathbf{x},-k \hat{\mathbf{z}}+\mathbf{y})-\frac{4 \pi h^{2}(z)}{k^{2}}\right]
\end{gathered}
$$

Setting the fluid velocity on the tube surface to zero results in equation (9)

$$
\int_{l(-1 / 2)}^{l(1 / 2)} d l(\mathrm{y}) \mathcal{M}_{z z}^{\prime}(\mathbf{x}, \mathbf{y}) f_{z}(\mathbf{y})+\int_{l(-1 / 2)}^{l(1 / 2)} d l(\mathrm{y}) \mathcal{M}_{z r}(\mathbf{x}, \mathbf{y}) f_{r}(\mathbf{y})+\left\{I(\mathbf{x})+4 \pi h^{2}\left(\frac{1}{2}\right)\right\}=0
$$

where

$$
I(\mathbf{x})=\int_{l(-1 / 2)}^{l(1 / 2)} d l(\mathbf{y}) \mathcal{M}_{z z}^{\prime}(\mathbf{x}, \mathbf{y}) z n_{z}(\mathbf{y})+\int_{l(-1 / 2)}^{l(1 / 2)} d l(\mathbf{y}) \mathcal{N}_{z z}^{\prime}(\mathbf{x}, \mathbf{y}) n_{z}(\mathbf{y})+\int_{l(-1 / 2)}^{l(1 / 2)} d l(\mathbf{y}) \mathcal{M}_{z r}(\mathbf{x}, \mathbf{y}) z n_{r}(\mathbf{y})+\int_{l(-1 / 2)}^{l(1 / 2)} d l(\mathbf{y}) \mathcal{N}_{z r}^{\prime}(\mathbf{x}, \mathbf{y}) n_{r}(\mathbf{y})
$$

A similar treatment for the $r$-component of equation (1) results in equation (10). The $r$-component of equation (1) is

$$
\int_{l(-1 / 2)}^{l(1 / 2)} d l(\mathbf{y}) \mathcal{M}_{r z}(\mathbf{x}, \mathbf{y}) f_{z}(\mathbf{y})+\int_{l(-1 / 2)}^{l(1 / 2)} d l(\mathbf{y}) \mathcal{M}_{r r}(\mathbf{x}, \mathbf{y}) f_{r}(\mathbf{y})+J(\mathbf{x})=0
$$

where,

$$
J(\mathbf{x})=\int_{l(-1 / 2)}^{l(1 / 2)} d l(\mathbf{y}) \mathcal{M}_{r z}(\mathbf{x}, \mathbf{y}) z n_{z}(\mathbf{y})+\int_{l(-1 / 2)}^{l(1 / 2)} d l(\mathbf{y}) \mathcal{N}_{r z}^{\prime}(\mathbf{x}, \mathbf{y}) n_{z}(\mathbf{y})+\int_{l(-1 / 2)}^{l(1 / 2)} d l(\mathbf{y}) \mathcal{M}_{r r}(\mathbf{x}, \mathbf{y}) z n_{r}(\mathbf{y})+\int_{l(-1 / 2)}^{l(1 / 2)} d l(\mathbf{y}) \mathcal{N}_{r r}(\mathbf{x}, \mathbf{y}) n_{r}(\mathbf{y})
$$

and

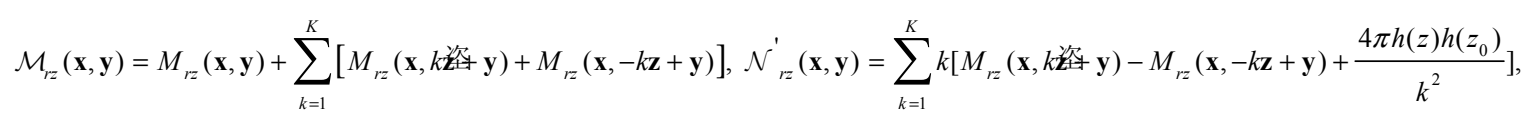

$$
\begin{aligned}
& \mathcal{M}_{r r}(\mathbf{x}, \mathbf{y})=M_{r r}(\mathbf{x}, \mathbf{y})+\sum_{k=1}^{K}\left[M_{r r}(\mathbf{x}, k+\mathbf{y})+M_{r r}(\mathbf{x},-k \mathbf{z}+\mathbf{y})\right], \mathcal{N}_{r r}(\mathbf{x}, \mathbf{y})=\sum_{k=1}^{K} k\left[M_{r r}(\mathbf{x}, k \text {; }\right.
\end{aligned}
$$


In order to solve equations (9) and (10) for obtaining components of surface force, the surface of the tube is discretized along the $z$-axis into $\mathrm{N}$ elements and the line integrals are approximated using the trapezoidal rule. Although the functions $M_{i j}(\mathbf{x}, \mathbf{y})$ are singular when $\mathbf{y}=\mathbf{x}$, the singularities are integrable in the sense of the Cauchy principal value. Therefore, the integrals are evaluated by subtracting the immediate neighborhood of the singular point from the integration domain, integrating the kernel analytically in the region, and then adding the result to the integral computed numerically over the remainder of the domain. For pressure drop $\Delta p$ across one wave section, integral equations (9) and (10) represent a linear system of $2 \mathrm{~N}$ algebraic equations for the $2 \mathrm{~N}$ unknown components of the force distribution on the tube surface, $\mathbf{f}=\left(f_{z}, f_{r}\right)$.

These equations are solved using an International Mathematics and Statistics Library (IMSL) routine. As described above, once the force on the tube surface is known, it can be used to the velocity distribution inside the tube domain.

\section{Results and Discussion}

We have presented our simulation results in this section. Figure 2 represents the profile of a sinusoidally constricted periodic tube in the upper region. We have analyzed our detailed velocity distribution in throat region and expansion region within this periodic tube. Figures 3-6 show the velocity profiles at different cross-sections for a fixed time while Figures 7-11 show the velocity profiles at different time for a fixed crosssection. In each figure, (a) shows the axial velocity profile whereas (b) shows the radial velocity profile. We have discussed the obtained results separately in the following two sections.

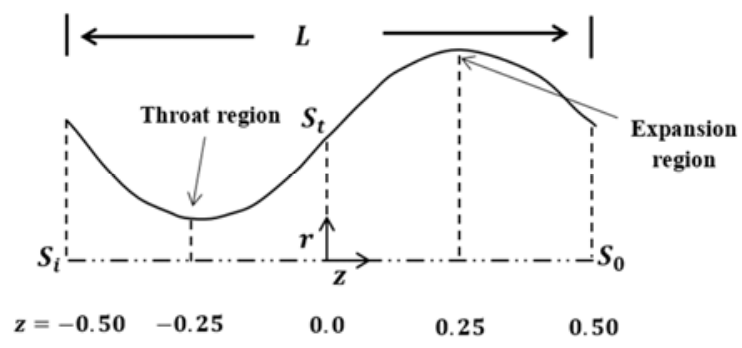

Figure 2. Profile of the sinusoidally constricted capillary.

\subsection{For Fixed Time at Different Cross Sections}

For the present oscillating flow, the profiles of the velocity field are computed and plotted in the figures for four different time periods $t=0.25, t=0.75, t=1.25, t=1.75$. We have found different similarities and dissimilarities among the figures which represent the velocity as well as the characteristics of the flow that we are working with. The axial velocity $\left(u_{z}\right)$ and radial velocity $\left(u_{r}\right)$ in the tube for different cross-sections at fixed time are represented in part "a" and "b", respectively. Five fixed values of $z$ are taken for five fixed cross-section and they are $z=-0.50, z=-0.25, z=0.00, z=0.25, z=0.50$. The cross-sections at $z=-0.50, z=0.50$ are both the converging region where as the cross-section at $z=0.00$ is known as diverging region. The constriction and expansion region are at the axial position $z=-0.25$ and $z=0.25$, respectively.

In Figure 3 (a), time $t=0.25$ is considered as fixed time and evaluated the values of axial velocity for different crosssections. It is found that, in the throat zone of the tube i.e. where $z=-0.25$, the tube has its minimum radius but has the maximum axial velocity for this cross-section. On the contrary, when the radius increases and reaches in its maximum value, i.e., in the expansion zone of the tube, where $z=0.25$, the velocity profile indicates the minimum axial velocity for this cross-section of the tube. The figure also shows the velocity profile for other three fixed values of $z$, where $z=-0.50, z=0.00$ and $z=0.50$. As the radii for all three crosssections are the same for the tube profile at this fixed time, it represents the same pattern of velocity for all cross-sections and axial velocity lies between velocity of the throat and expansion zone of the tube. Figure 5 (a) shows the axial velocity profile when time $t=1.25$ is fixed. However, it visualizes the same pattern like Figure 3 (a). Since we consider oscillating flow for calculating velocity, we obtain the same velocity pattern at a fixed cross-section after completing a full oscillation and so when $t=1.25$ is regarded as fixed time, it indicates the completion of an oscillation after considered first fixed time $t=0.25$. After observing both Figures 3 (a) and 5 (a), it is stated that, velocity is inversely proportional to the radius of the tube as well as it remains the same at a fixed cross-section after a full oscillation. Now move on the Figure 4 (a) that represents the axial velocity profile at different crosssections when time $t=0.75$ and it shows the same patterns but in opposite direction to the velocity when time $t=0.25$ and $t=1.25$ which are depicted in Figures 3 (a) and 5 (a).

Here, the maximum velocity occurs at $z=-0.25$ but in opposite direction when radius is minimum i.e. throat region of the tube. In the case of $z=0.25$ i.e. the expansion region of the tube when radius is maximum, minimum axial velocity occurs. As before, for the cross-section $z=-0.50, z=0.00$ and $z=0.50$, the axial velocity remains between the velocities occurred for expansion $(z=0.25)$ and throat $(z=-0.25)$ region of the tube. The behaviors of the curves in Figure 6 (a) are the same as in Figure 4 (a), since time $t=1.75$ is considered as a fixed time which indicates the completion of a full oscillation after time $t=0.75$. In a nutshell, axial velocity for a tube profile is inversely proportional to the radius of its cross-section and indicates the same pattern at a fixed cross-section after each full oscillation. In each of the above cases, the axial velocity decreases with increasing radius at a fixed cross-section.

Radial velocity profiles are shown in part " $b$ " in every figure. Figure 3 (b) represents that the radial velocity is zero for the expansion and throat region of the tube when time $t=0.25$ is considered as fixed time. Both the radial velocity profiles at the cross-section $z=0.25$ and $z=-0.25$ are lied on the zero line and consequently there is no change of radial velocity along the whole radius. But, radial velocity changes with respect to its radius for the cross-section $z=-0.50, z=0.00$ and $z=0.50$ due to converging and diverging zones. The radial velocity profiles at the cross-section $z=-0.50$ and $z=0.50$ are the same since both 
cross-sections are in the converging zone. On the contrary, the radial velocity for the cross-section $z=0.00$ are the same but in opposite direction to the radial velocity at the cross-section $z=-0.50$ and $z=0.50$, since the cross-section at the axial position $z=0.00$ is in the diverging region. Similarly, due to oscillating flow, exactly the same radial velocity curves as in Figure 3 (b) are shown for Figure 5 (b) where we consider fixed

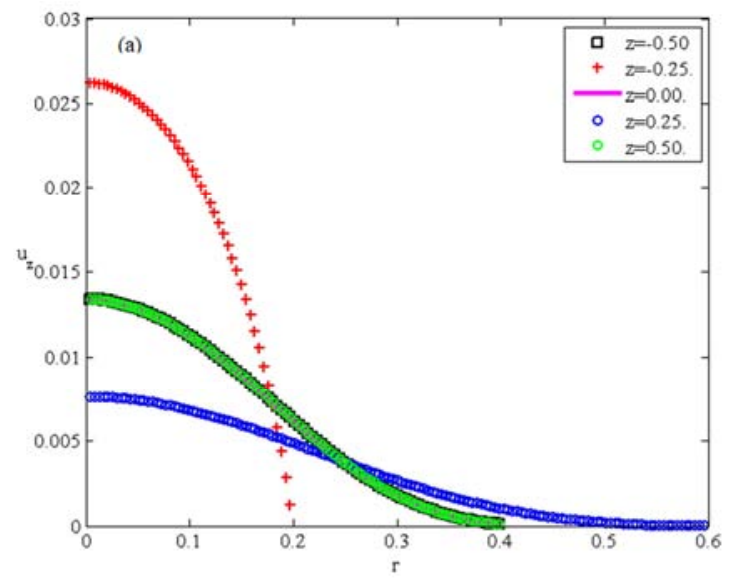

time $t=1.25$. Figures 4 (b) and 6 (b) represents the radial velocity for the time $t=0.75$ and $t=1.75$, respectively. The same radial velocity but in opposite direction occurs in both figures at the converging zones at $z=-0.50, z=0.50$ and at the diverging zone at $z=0.00$. Interestingly as before, there is also no radial velocity for both the cross-sections at $z=0.25$ and $z=-0.25$.

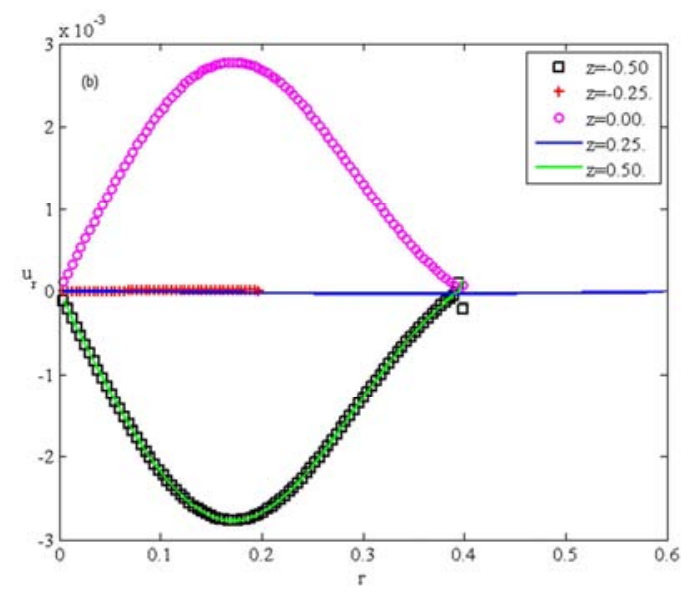

Figure 3. (a) The axial velocity profile and (b) the radial velocity profile in upper portion of the tube for the cross-sections $z=-0.50, z=-0.25, z=-0.00, z=0.25, z=0.50$ at time $t=0.25$.
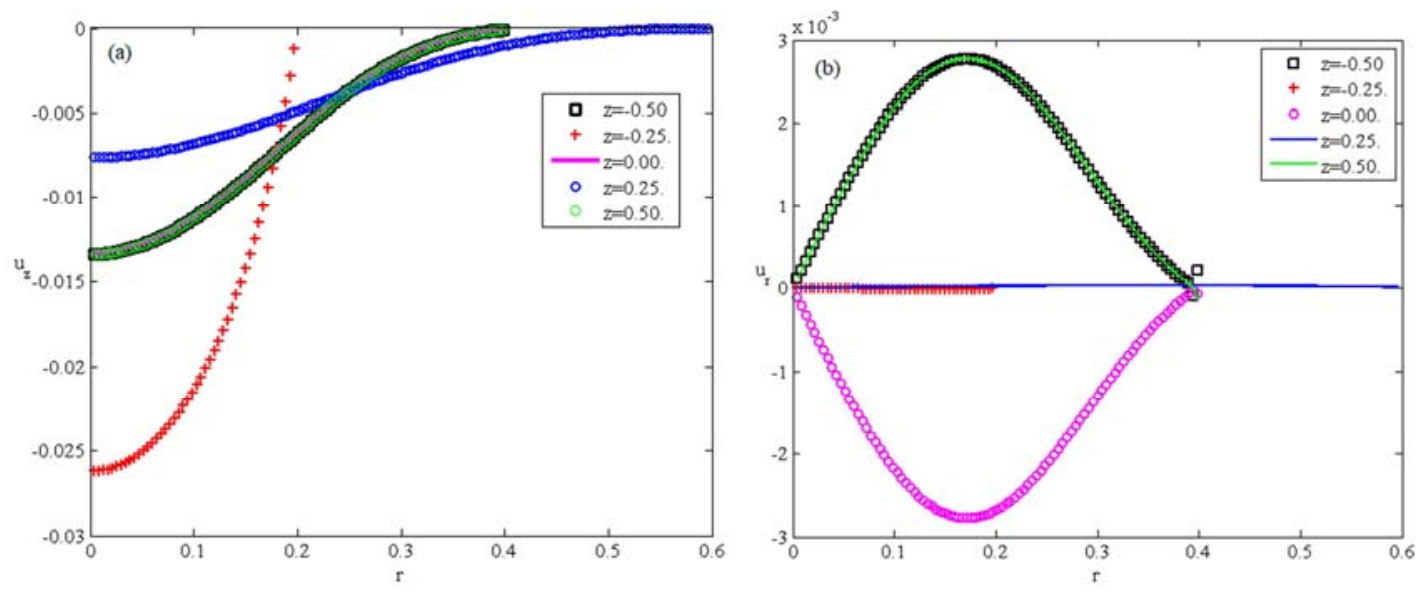

Figure 4. (a) The axial velocity profile and (b) the radial velocity profile in upper portion of the tube for the cross-sections $z=-0.50, z=-0.25, z=-0.00, z=0.25, z=0.50$ at time $t=0.75$.
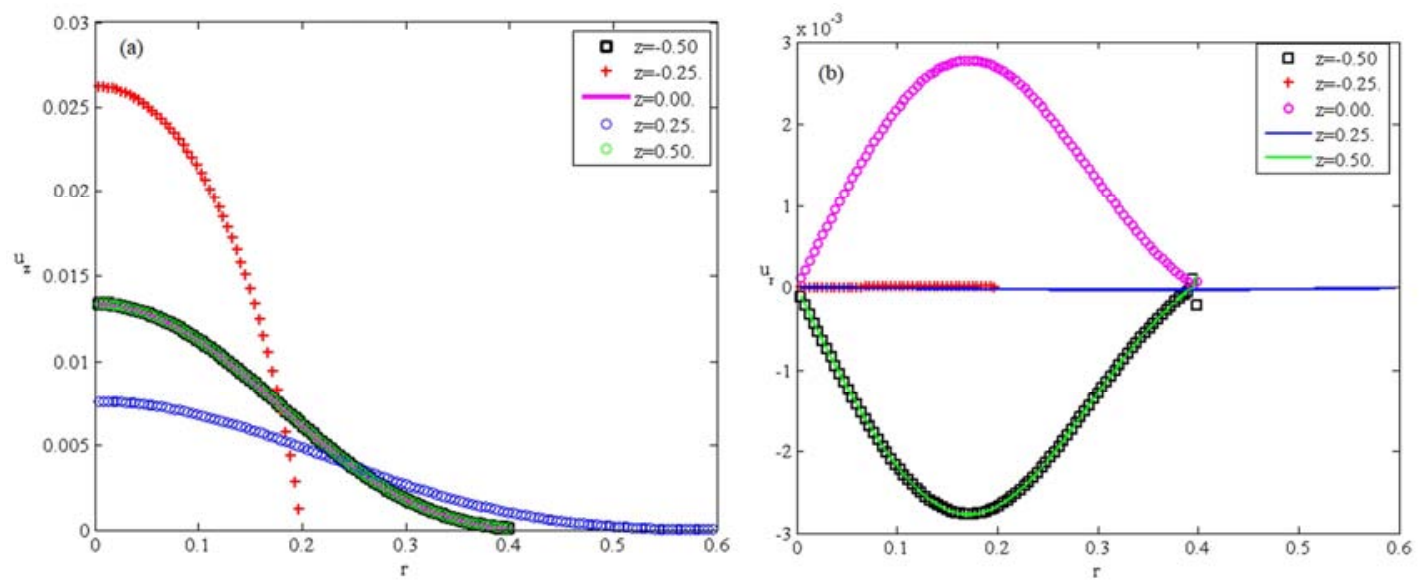

Figure 5. (a) The axial velocity profile and (b) the radial velocity profile in upper portion of the tube for the cross-sections $z=-0.50, z=-0.25, z=-0.00, z=0.25, z=0.50$ at time $t=1.25$. 

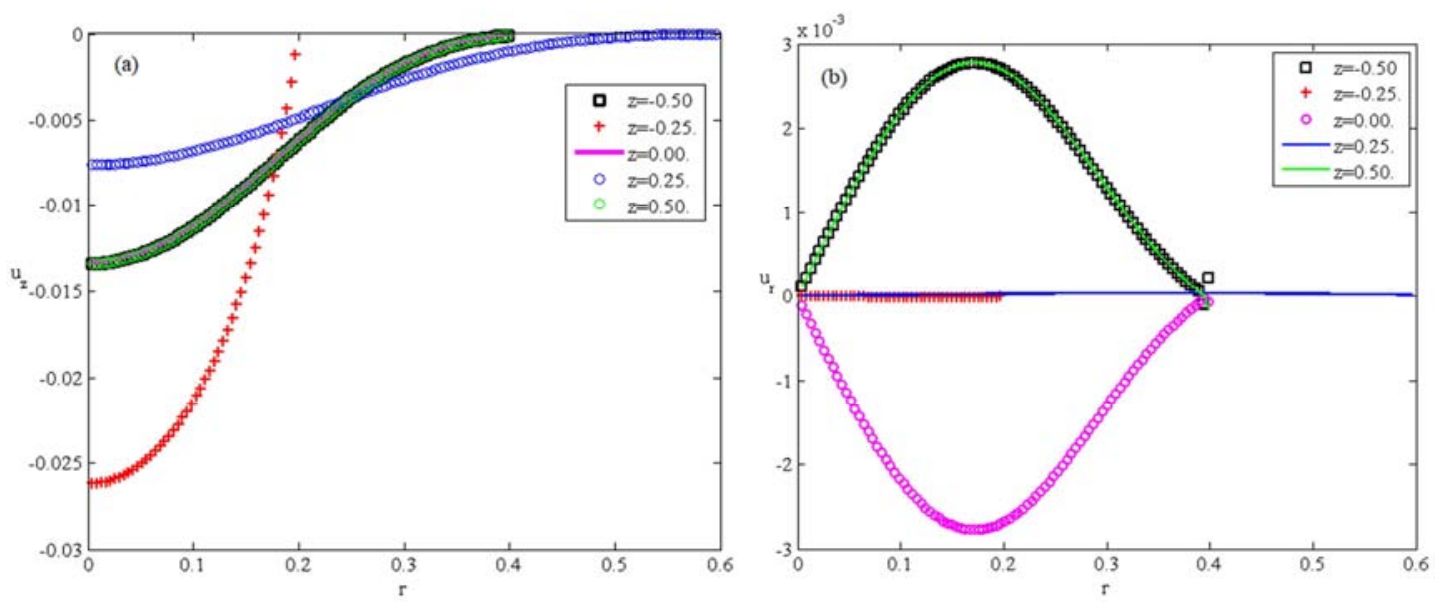

Figure 6. (a) The axial velocity profile and (b) the radial velocity profile in upper portion of the tube for the cross-sections $z=-0.50, z=-0.25, z=-0.00, z=0.25, z=0.50$ at time $t=1.75$.

\subsection{For Fixed Cross Section at Different Times}

Velocity profiles for a fixed cross section at different time are presented in this section. Firstly, we fixed the cross section $z=-0.50$ for different time $t=0.25, t=0.75, t=1.00, t=1.25, t=1.75$, and $t=1.75$ and checked the velocity profiles of the fluid. Figure 7 (a) illustrated the behavior of the axial velocity profile at a specific axial position $z=-0.50$ for different time $t=0.25, t=0.75, t=1.00, t=1.25, t=1.75$. This figure depicts that the axial velocity for the time $t=0.25, t=1.25$ and $t=0.75, t=1.75$ are the same in magnitude but in opposite direction. Interestingly, there is no axial velocity for time $t=1.00$ since the pressure difference is zero at this instant of time. Figures 9 (a) and 11 (a) show axial velocity for the cross-section $z=0.00, z=0.50$ respectively and indicate the same axial velocity pattern with respect to Figure 7 (a). Considering the axial velocity profile for the cross-section $z=0.25$, we also obtain the same pattern as before (Figures 7 (a), 9 (a), 11 (a)) but the magnitudes of velocity are smaller than before (Figures 7 (a), 9 (a), 11 (a)) for the time both $t=0.25, t=1.25$ and $t=0.75, t=1.75$ whereas the line describes

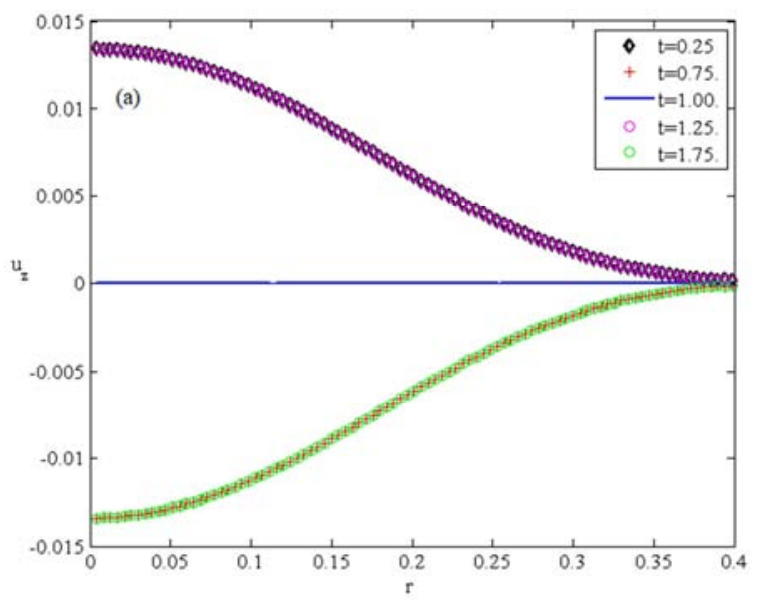

the velocity for $t=1.00$ remains unchanged (see Figure 10 (a)). However, slightly different pattern can be seen in Figure 8 (a). Although the axial velocity for $t=0.25, t=1.25$ and for $t=0.75, t=1.75$ are the same in magnitude but in opposite direction as before. Radial velocity for cross-section $z=-0.50$ is shown in Figure 7 (b) and it represents that the radial velocity for $t=0.25, t=1.25$ and for $t=0.75, t=1.75$ are the same in magnitude but in opposite direction. For $t=1.00$ we get a zero-radial velocity since the pressure difference is zero at this time. By observing Figures 7 (b) and 11 (b), we obtain the same radial velocity profiles for $z=-0.50$ and $z=0.50$ because both are the converging zones of the tube profile where flow entered in. On the other side, Figure 9 (b) shows the same radial velocity in magnitude but in opposite direction as in the radial velocity in Figure 7 (b) and 11 (b), since $z=0.00$ is the axial position in digerging zone where the fluid spread out. Interestingly, the radial velocity profiles in Figures 8 (b) and 10 (b) are not only different in magnitude but also different in direction. It is noted from Figure 8 (b) that the radial velocity increases linearly with increasing radius, which is distinctive trends compared to radial velocity at other cross-sections.

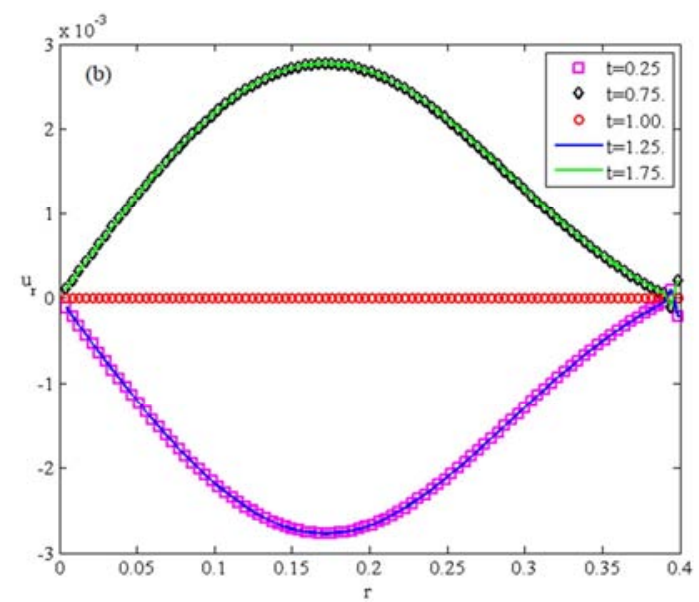

Figure 7. (a) The axial velocity profile and (b) the radial velocity profile for different time $t=0.25, t=0.75, t=1.00, t=1.25, t=1.75$ at the cross-section $z=-0.50$. 

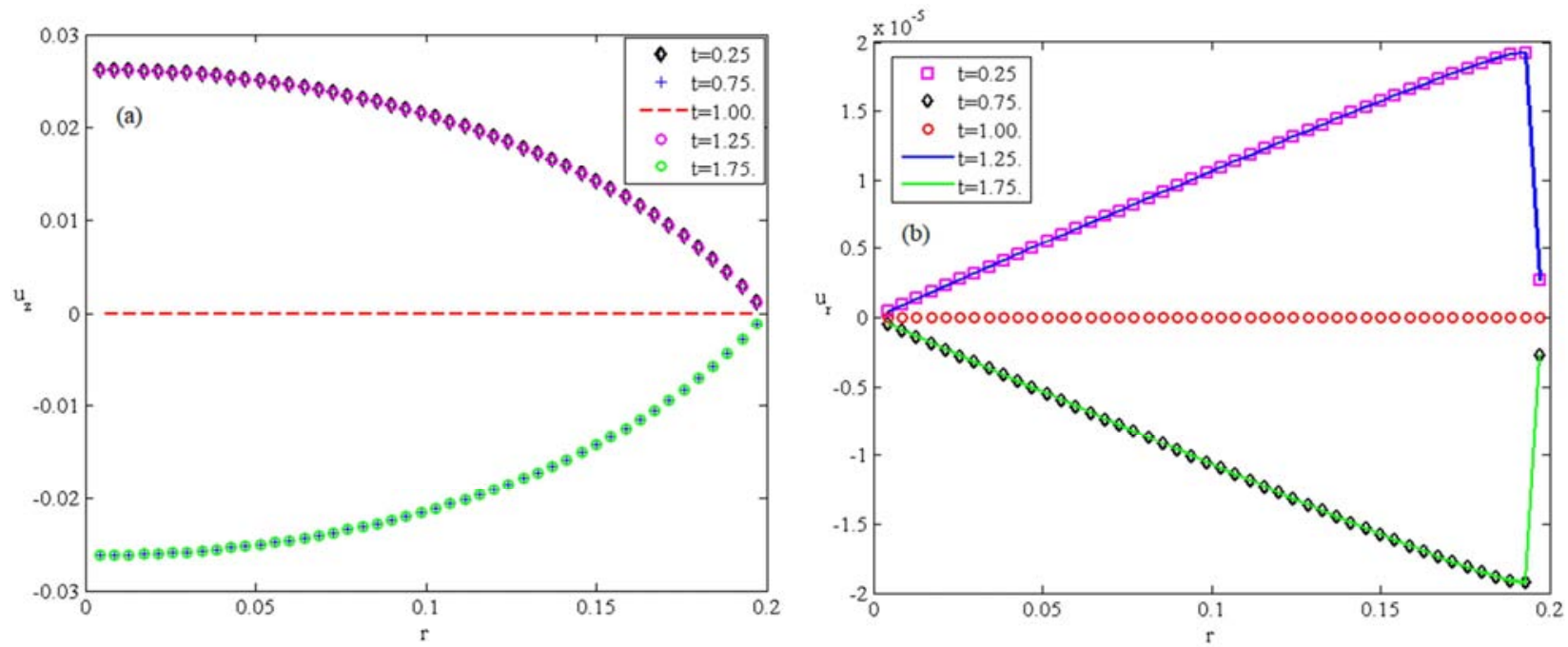

Figure 8. (a) The axial velocity profile and (b) the radial velocity profile for different time $t=0.25, t=0.75, t=1.00, t=1.25, t=1.75$ at the cross-section $z=-0.25$.
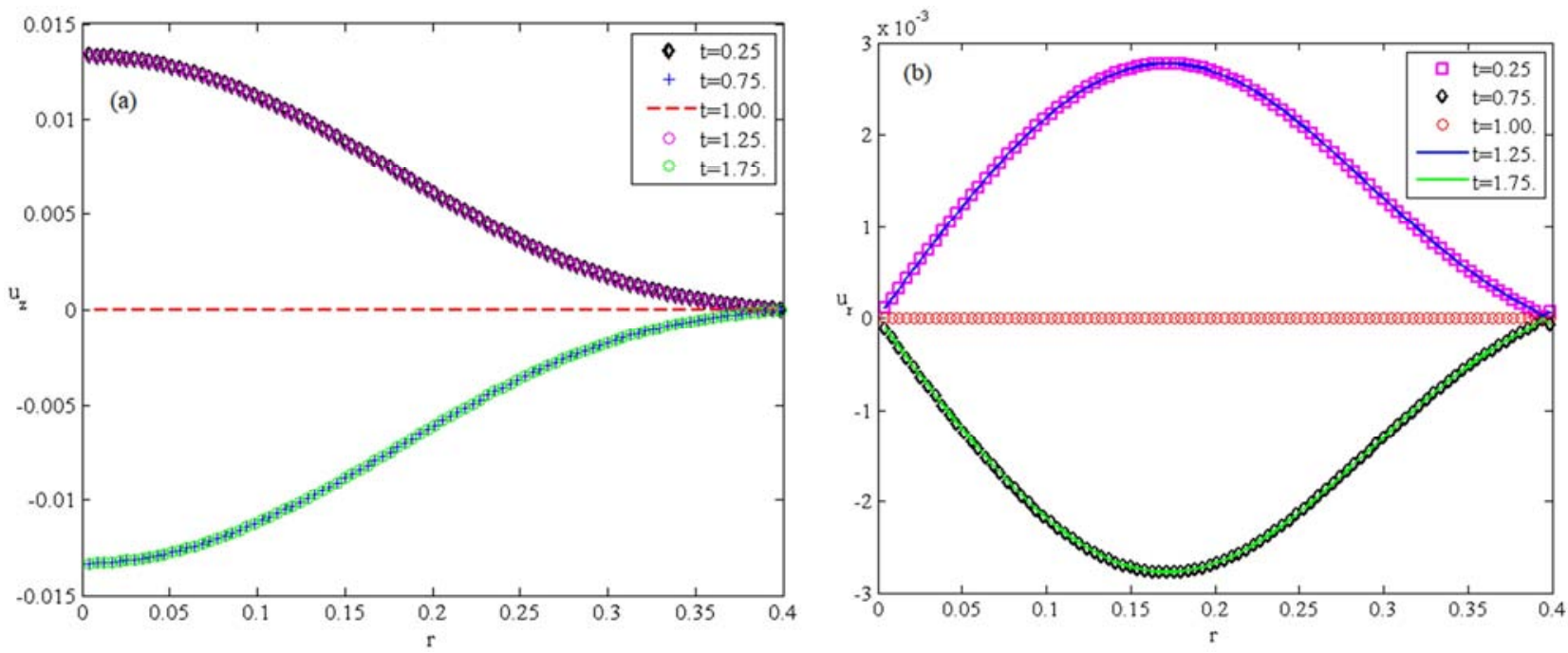

Figure 9. (a) The axial velocity profile and (b) the radial velocity profile for different time $t=0.25, t=0.75, t=1.00, t=1.25, t=1.75$ at the cross-section $z=0.00$.
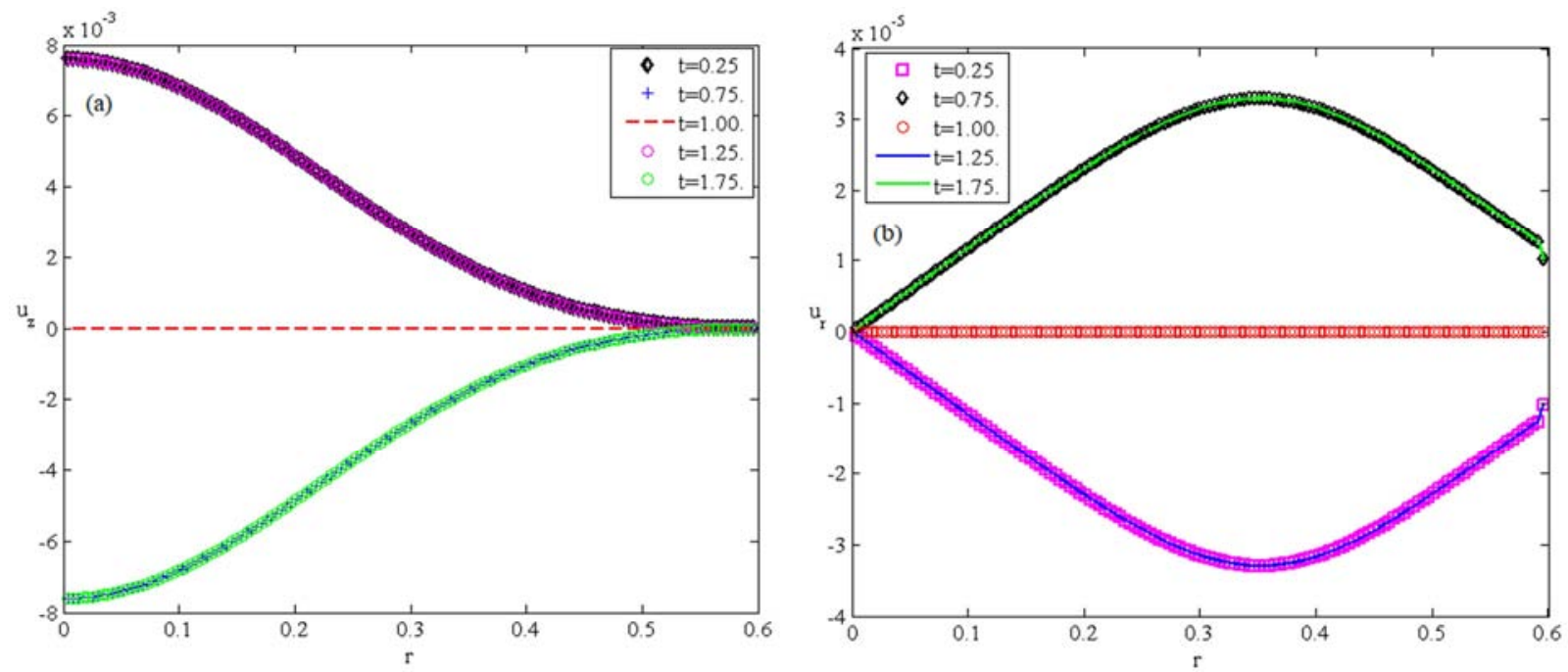

Figure 10. (a) The axial velocity profile and (b) the radial velocity profile for different time $t=0.25, t=0.75, t=1.00, t=1.25, t=1.75$ at the cross-section $z=0.25$. 

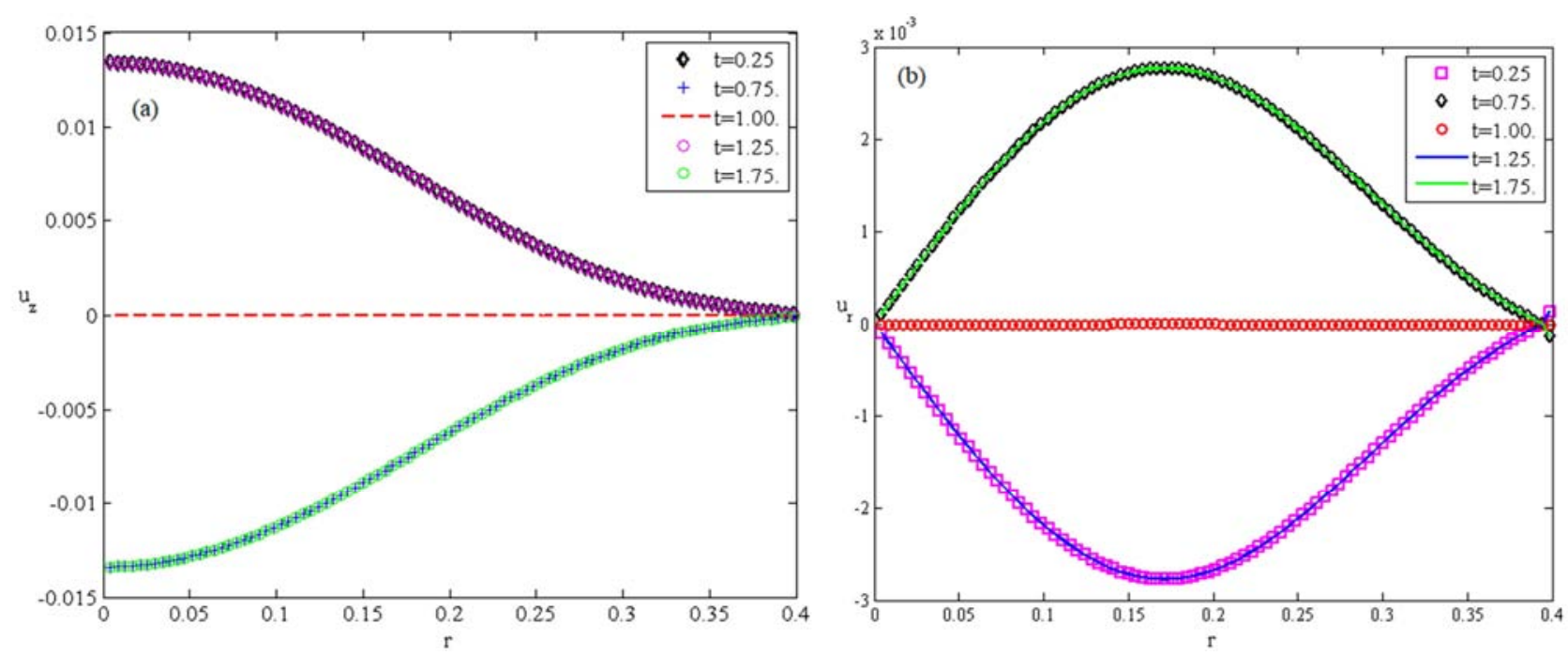

Figure 11. (a) The axial velocity profile and (b) the radial velocity profile for different time $t=0.25, t=0.75, t=1.00, t=1.25, t=1.75$ at the cross-section $z=0.50$.

From the above mentioned observation, we find that the behaviors of the velocity curves for different cross-section remain the same for the same phase of time over the oscillation of flow which expresses that pattern of curves remains similar for either $t=0.25$ and $t=1.25$ or $t=0.75$ and $t=1.75$. On the contrary, behavior of the velocity curves becomes different for different phase of time. Also, the axial velocity at the converging and diverging regions are the same while the radial velocity at these regions are the same in magnitudes but in opposite direction. Finally, the radial velocity is maximum in the half way between the tube axis and the tube wall, and it is minimum on the tube axis and on the tube wall.

\section{Conclusions}

In this paper, we have investigated oscillating flow of viscous incompressible fluid through sinusoidal periodic tube at low Reynolds number in order to investigate the effectiveness of the flow through periodic cross-section of tube shape on the particle separation process. Due to investigate the fluid movement through a distinct wavesection, we have used the boundary element method (BEM) to solve the governing equations, which is helpful to examine the fluid velocity numerically. After solving the governing equations, we have calculated the components of the surface force on the tube wall and obtained the axial and radial velocities at different cross-sections and for different time, and found out the relation between them. Firstly, we investigate axial and radial velocity for different crosssection at a fixed time where we study the velocity at five different cross-sections for four different times. We found the inversely proportional relationship between the axial velocity and the radius at each cross-section. The radial velocity remains the same at either converging or diverging zone for a fixed time. We also observed the effect of both axial and radial velocity for four different time at those five fixed cross-sections. We have found the same velocity curves for the same phase of time whereas the curves show different behavior for different phase of time over the oscillation. Moreover, the axial velocity also remains the same at the conversing and diverging region while the radial velocity are the same in magnitudes but in opposite direction at that region. The radial velocity is maximum and minimum at the half way and the tube axis or wall, respectively. In a nutshell, we can say that the characteristics of velocity profiles at different cross-sections remain the same for a fixed time due to the geometry of the periodic tube. Also, the velocity profiles, as expected, at a specific cross-section are similar after each complete oscillation. The obtained results indicate that there is no net movement of the fluid particle after each complete oscillation, which is considered as an assumption that use in the particle separation process.

\section{References}

[1] Taylor, L. A. and Gerrard, J. H. (1977) Pressure-radius relationships for elastic tubes and their application to arteries: Part 1. Theoretical relationships, Medical and Biological Engineering and Computing 15 (5), 11-17.

[2] Jawadzadegan, A., Esmaeili, M., Majidi, S. and Fakhimghanbarzadeh, B. (2009) Pulsatile flow of viscous and viscoelastic fluids in constricted tubes, J. Mech. Sci. Technol 23, 2456-2467.

[3] Hewitt, G. F. and Marshall, J. S. (2010) Particle focusing in a suspension flow through a corrugated tube, J. Fluid Mech. $660,258-281$.

[4] Fedkiw, P. and Newman, J. (1977) MasstransferathighPecletnumbersforcreepingflow in a packed-bed reactor, AIChE J. 23, 255-263.

[5] Kettner, C., Reimann, P., Hanggi, P. and Muller, F. (2000) Drift ratchet, Phys. Rev. E 61, 312-323.

[6] Matthias, S. and Muller, F. (2003) Asymmetric pores in a silicon membrane acting as massively parallel Brownian ratchets, Lett. Nat. 424, 53-57. 
[7] Bellhouse, B. J., Bellhouse, F. H., Curl, C. M., MacMillan, T. I., Gunning, A. J., Spratt, E. H., MacMurray, S. B. and Nelems, J. M. (1973) A high efficiency membrane oxygenator and pulsatile pumping system and its application to animal trials, Trans. Am. Soc. Artif. Intern. Organs 19, 72.

[8] Sobey, I. J. (1980) On flow through furrowed channels. Part 1. Calculated flow patterns, J. Fluid Mech. 96, 1.

[9] Sobey, I. J. (1983) The occurrence of separation in oscillatory flow, J. Fluid Mech. 134, 247.

[10] Stephanoff, K. D., Sobey, I. J. and Bellhouse, B. J. (1980) On flow through furrowed channels. Part 2. Observed flow patterns, J. Fluid Mech. 96, 27.

[11] Nishimura, T., Ohori, Y. and Kawamura, Y. (1984) Flow characteristics in a channel with symmetric wavy wall for steady flow, J Chem Eng Japan, 17, 466-471.

[12] Nishimura, T. and Kojima, N. (1995) Mass transfer enhancement in a symmetric sinusoidal wavy-walled channel for pulsatile flow, Int. J. Heat Mass Transfer 38, 1719-1731.
[13] Ralph, M. E. (1986) Oscillatory flows in wavy-walled tubes, J. Fluid Mech. 168, 515.

[14] Lee, B. S., Kang, I. S. and Lim, H. C. (199) Chaotic mixing and mass transfer enhancement by pulsatile laminar flow in an axisymmetric wavy channel, International Journal of Heat and Mass Transfer 42, 2571-2581.

[15] Nishimura, T., Bian, Y. N., Kunitsugu, K. and Morega, A. M. (2003) Fluid flow and mass transfer in a sinusoidal wavywalled tube at moderate Reynold numbers, Heat TransferAsian Research 7, 650-661.

[16] Chakravarty, S. and Sen, S. (2006) A mathematical model of blood flow and convective diffusion processes in constricted bifurcated arteries, Korea-Australia Rheology Journal 18, 51-65.

[17] Islam, N., Bradshaw-Hajek, B. H., Miklavcic, S. J. and White, L. R. (2015) The onset of recirculation flow in periodic capillaries: Geometric effects, European Journal of Mechanics B/Fluids 53, 119-128.

[18] Pozrikidis, C. (1992) Boundary Integral and singularity Methods for Linearised Viscous Flow, Cambridge University Press, Cambridge, U. K. 